\title{
Improved Water Injection Index Curve on Estimation of Oil in Place for Fractured Vuggy Carbonate Reservoirs
}

\author{
Fan Haijun, ", Kang Zhijiang ${ }^{2}$, Zheng Songqing ${ }^{2}$, Zhu Guiliang ${ }^{2}$ \\ ${ }^{1}$ School of Petroleum Engineering, China University of Petroleum, Qingdao, China \\ ${ }^{2}$ Petroleum Exploration \& Production Research Institute, SINOPEC, Beijing, China \\ Email address: \\ petroengineer@126.com (Fan Haijun), kangzj.syky@sinopec.com (Kang Zhijiang), zhengsq.syky@sinopec.com (Zheng Songqing), \\ zhugl.syky@sinopec.com (Zhu Guiliang) \\ *Corresponding author
}

\section{To cite this article:}

Fan Haijun, Kang Zhijiang, Zheng Songqing, Zhu Guiliang. Improved Water Injection Index Curve on Estimation of Oil in Place for Fractured Vuggy Carbonate Reservoirs. International Journal of Oil, Gas and Coal Engineering. Vol. 8, No. 6, 2020, pp. 124-129. doi: 10.11648/j.ogce.20200806.11

Received: October 15, 2020; Accepted: October 26, 2020; Published: November 4, 2020

\begin{abstract}
Fractured vuggy carbonate reservoirs play an important role in the world crude oil resources. The significant reservoir heterogeneity represented by complex distribution of multi-scale fractures and vugs brings challenges to reservoir oil in place (OIP) estimation, which is a fundamental parameter in oil field development. OIP estimation based on production dynamic analysis is regularly carried out in oilfield practice, the result of which is normally adopted as a comparison to volumetric calculation from geological study for mutual verification. This paper introduces a widely used water injection index curve method on OIP estimation of fractured vuggy carbonate reservoir in Tarim oilfield, which is a straight forward plot of bottom-hole pressure versus cumulative water injection. An improved model taking into consideration the compressibility of second gas gap is presented and applied on real well from Tahe carbonate reservoir. The application of the new model demonstrated that conventional method tends to over-estimate the OIP, while the improved model gives a relatively reliable estimation. The improved water injection index curve in this paper is easy to be conducted based on well production and injection dynamic and shows advantages on convenient pressure conversion from well-head to well-bottom and avoiding dealing with the high uncertainty of aquifers in carbonate reservoir.
\end{abstract}

Keywords: Fractured Vuggy Carbonate Reservoir, Oil in Place, Water Injection Index Curve, Oil Compressibility

\section{Introduction}

Fractured vuggy carbonate reservoirs contribute a large proportion of world oil resources, characterized by high heterogeneity and complex fracture-vug/cave system. Figure 1 shows a schematic diagram of fractured vuggy reservoir from Tarim oilfield, in which fractures and multi-scales vugs or caves provide storage and flow channels for reservoir fluids while the matrix system is impermeable and does not contribute for production $[1,2]$. Reservoir oil in place (OIP) estimation in fractured carbonate reservoir is always big challenge and of great importance in the oilfield development.

Serval methodologies have been proposed to estimate well-controlled oil in place, such as well test, flowing material balance equation, or production transient analysis
[3-5]. Different methods requires different data and have different advantages and drawbacks [6,7]. Due to the high formation discontinuity and strong stochastic distribution of reservoir parameters, OIP estimation for fractured-vuggy carbonate reservoirs requires exclusive techniques considering the available dynamic performance data.

OIP estimation based on production dynamic analysis is commonly applied in oilfield practice, the result of which is normally adopted as a comparison to geological study for mutual verification.

How to effectively use the dynamic field production data, such as the high frequency production and injection data, wellhead pressure, becomes a task of successful management of fractured vuggy carbonate reservoirs [8-11, 15-16].

Most of the carbonate reservoirs in Tarim oilfield have conducted water injection or gas injection for different 
purposes, such as energy supplementation or water coning mitigation. The dynamic performance analysis in fractured vuggy carbonate reservoir requires new methods or techniques compared to conventional sandstone reservoirs.

This paper introduces the commonly adopted water injection index curve method in reservoir dynamic analysis and provides an improved model considering the impact of secondary gas cap. The application of the new model in Tahe fractured-vuggy carbonate reservoir illustrated the accuracy and improvement in OIP estimation.

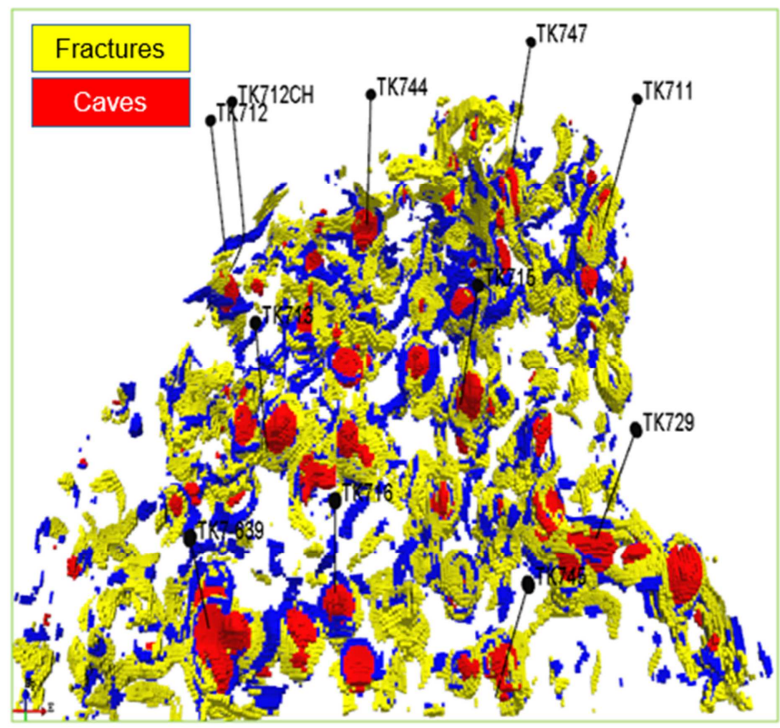

Figure 1. Schematic Diagram of Fractured Vuggy Reservoirs.

\section{Methodologies}

\subsection{Conventional Water Injection Index Curve}

For water injection wells the plot of bottom-hole pressure versus water injection rate normally reflects the injectivity or variation of well conditions. Meanwhile in fractured vuggy reservoirs, reservoir engineers discovered more potential applications of this slope analysis, such as formation type verification and injection-parameter optimization $[12,13]$.

Suppose a volumetric fractured vuggy reservoir with a well penetrating one of the caves, in the water injection process, the volume change of oil is equivalent to cumulative water injection if neglecting the compressibility of reservoir rock and water.

The volume change of oil is:

$$
\Delta V=V_{o}-V_{o}^{\prime}=V_{w}
$$

From the definition of oil compressibility,

$$
C_{o}=\frac{1}{V_{o}} \frac{\Delta V_{o}}{\Delta p}
$$

In which $V_{\mathrm{o}}$ is the oil in place, $V_{\mathrm{w}}$ is cumulative water injection, $\Delta p=p_{\mathrm{i}}-p$ is the pressure change after water injection,
From (1) and (2), a linear expression can be found:

$$
p=\frac{1}{C_{o} V_{o}} V_{w}+p_{i}
$$

From equation (3) it is clear that a plot of injection pressure vs cumulative water injection shows a straight line and the oil in place can be determined from the slope if oil compressibility is given. This is the foundation of conventional water injection index curve. And the pressure $p$ in equation (3) can be replaced approximately by well-head pressure for convenience.

Serval types of water injection index curve are shown in Figure 2, in which (a) is the normal plot, (b) means there are two connected caves around the well and the oil in place of the second cave can also be calculated from the two slopes. As a well may conduct serval round of water injection operations, the slope will increase for each round of water injection, indicating a reduced oil in place, as shown in Figure 2 (c). For the curves with same slope in (d) of Figure 2 , different intercept refers to the different initial injection pressure.

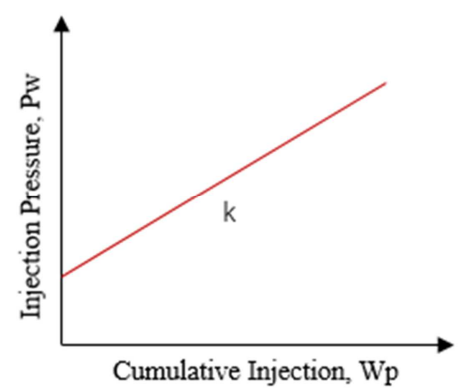

(a)

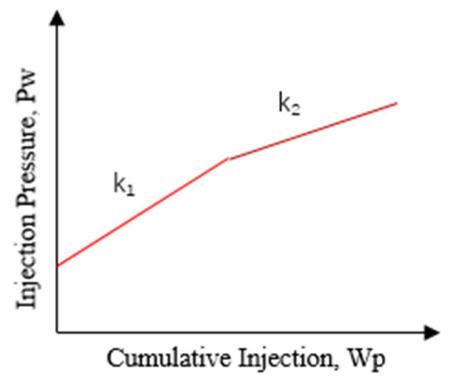

(b)

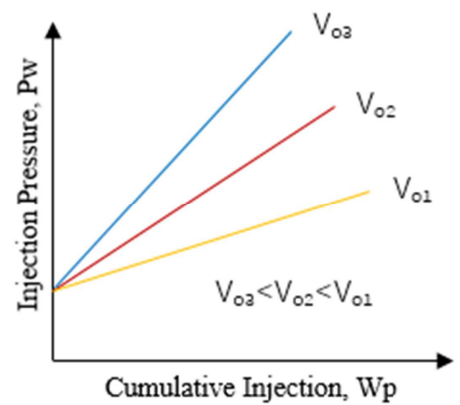

(c) 


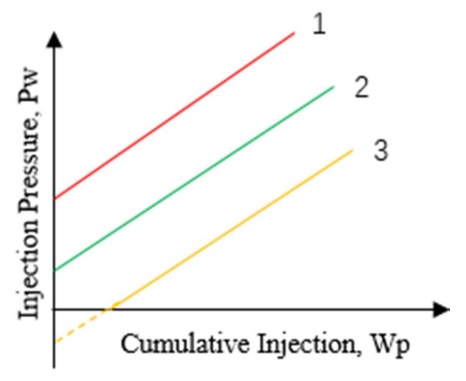

(d)

Figure 2. Various Types of Water Injection Index Curve.

This method requires only injection pressures and rate data, which are routinely collected in carbonate reservoir water injection with no interruption of regular field operations. It provides an efficient and simple method for OIP evaluation and has been applied in some of the wells in Tarim oilfield.

\subsection{Improved Water Injection Index Curve Model}

For the purpose of producing "attic oil", some fractured vuggy carbonate reservoirs have conducted gas injection operations to displace up-structure remaining oil. On the other hand, for some reservoirs with high gas-oil ratio, the solution gas will be released when the pressure drop down below to bubble point pressure. Actually a large volume of gas will be liberated near the well bottom even with the average reservoir pressure still above bubble point pressure. Due to the density difference some of the liberated solution gas will migrate up to the top of the reservoir structure and forms a secondary gas cap, shown in Figure 3. The compressibility of the second gas cap cannot be neglected in the water injection process.

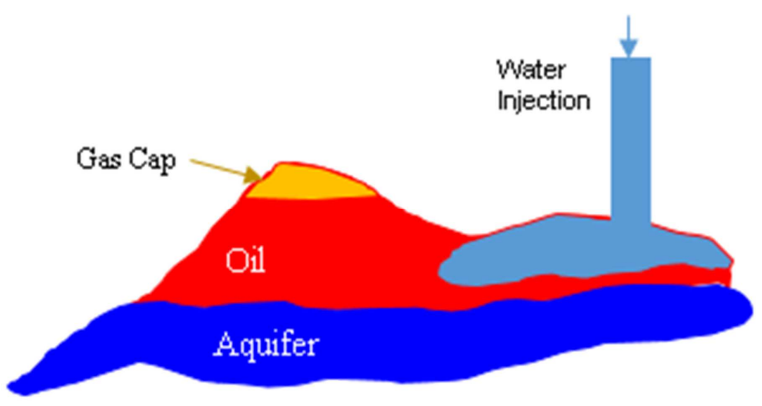

Figure 3. Water Injection When Secondary Gas Cap Exists.

Assume $V_{\mathrm{g}}$ is the volume of the second gas cap, which can be expressed by:

$$
V_{g}=\left[N R_{s i}-N_{p} R_{p}-\left(N-N_{p}\right) R_{s}\right] B_{g}
$$

After water injection, the reservoir pressure increases from $p$ to $p$ ' and the gas cap volume becomes $V_{\mathrm{g}}$,

$$
V_{g}{ }^{\prime}=\left[N R_{s i}-N_{p} R_{p}-\left(N-N_{p}\right) R_{s}\right] B_{g}{ }^{\prime}
$$

The gas formation volume factor can also be expressed as,

$$
B_{g}=\frac{T P_{S C}}{T_{S C}} \frac{z}{p}
$$

So the volume change of gas cap is,

$$
\begin{aligned}
& \Delta V_{g}=V_{g}-V_{g} \\
& =\left[N\left(R_{s i}-R_{s}\right)-N_{p}\left(R_{p}-R_{s}\right)\right] \frac{T P_{S C}}{T_{S C}}\left(\frac{z}{p}-\frac{z^{\prime}}{p^{\prime}}\right)
\end{aligned}
$$

As previously described, the volume change of oil in place before and after water injection is,

$$
\Delta V_{o}=C_{o} \Delta p V_{o}=C_{o} \Delta p\left(N-N_{p}\right) B_{o}
$$

The injected water volume is approximately equal to the total volume change of oil and gas, when neglecting reservoir rock and water compressibility.

$$
\Delta V_{g}+\Delta V_{o}=V_{w}
$$

Substitute (7) and (8) into (9), it gives,

$$
\begin{aligned}
& {\left[N\left(R_{s i}-R_{s}\right)-N_{p}\left(R_{p}-R_{s}\right)\right] \frac{T P_{S C}}{T_{S C}}\left(\frac{z}{p}-\frac{z^{\prime}}{p^{\prime}}\right)} \\
& +C_{o} \Delta p\left(N-N_{p}\right) B_{o}=V_{w}
\end{aligned}
$$

A final expression about $N$ can be derived from (10),

$$
N=\frac{V_{w}+C_{o} \Delta p N_{p} B_{o}+N_{p}\left(R_{p}-R_{s}\right) \frac{T P_{S C}}{T_{S C}}\left(\frac{z}{p}-\frac{z^{\prime}}{p^{\prime}}\right)}{C_{o} \Delta p B_{o}+\left(R_{s i}-R_{s}\right) \frac{T P_{S C}}{T_{S C}}\left(\frac{z}{p}-\frac{z^{\prime}}{p^{\prime}}\right)}
$$

The oil in place $N$ will keep constant during water injection, so if we draw a plot of $N$ versus cumulative water injection $V_{\mathrm{w}}$, the curve will appear to be a horizontal line. Then oil in place can be determined from the value of this horizontal line. This is the improved technique to evaluate oil in place through water injection index curve. Compared with this new method, the conventional method in section 2.1 tends to over-estimate oil in place because of neglecting the impact of secondary gas cap.

\section{Application}

\subsection{Use of Conventional Water Injection Index Curves}

T6-433CX is an oil well from the typical fractured vuggy carbonate reservoir in Tahe oilfield. This well, with a history of eight years, has undergone high daily oil production in the first two years then suffered from rapid production decline and severe bottom water coning problem. The dramatic water-cut fluctuations shown in Figure 4 indicts that there was strong water-coning problem from the bottom aquifer, leading to frequent well shut-ins when the water-cut reaches $100 \%$. 
Production restored again after water level stabilized depending on gravity segregation. In July 2007 and April 2008 this well conducted two water injection operation to mitigate the water coning. The injection data are list in the first four columns of Table 1.

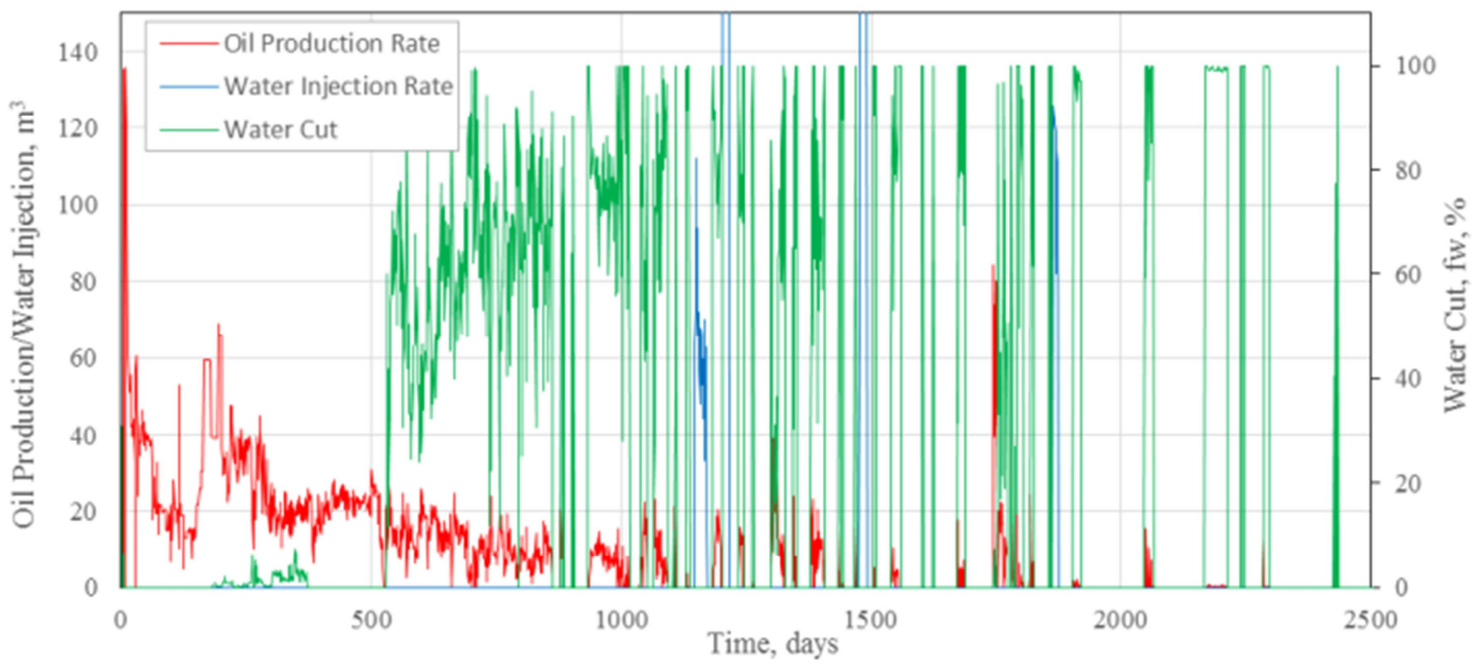

Figure 4. Daily Production Performance of Well T6-433CX.

According to conventional water injection index curve, a plot of bottom-hole pressure versus cumulative injection is drawn, as shown in Figure 5, and the straight-line equation is:

$$
p=0.0017 V_{\mathrm{w}}+59.669
$$

From fluid property study, the oil compressibility is $0.001276 \mathrm{MPa}^{-1}$, so the well-controlled oil in place is
$46.10 \times 10^{4} \mathrm{~m}^{3}$.

According to geological study, the controlled area of T6-433CX is $0.17 \mathrm{~km}^{2}$, the volumetric oil in place is $8.5 \times 10^{4}$ ton, which is $10.47 \times 10^{4} \mathrm{~m}^{3}$. It is noted that the oil in place from conventional water injection index curve is much high than that from geological study, so further investigation must be conducted.

Table 1. Water Injection Relevant Data of Well T6-433CX.

\begin{tabular}{|c|c|c|c|c|c|c|}
\hline Date & Qw m $3 / d$ & Pwf MPa & $V w \mathbf{m}^{3}$ & $\triangle \mathrm{P}$ MPa & $\mathbf{Z}$ & $\mathrm{N} \mathrm{m}^{3}$ \\
\hline $2008 / 04 / 14$ & 455 & 61.35 & 980 & 1.68 & 1.300 & 330257 \\
\hline $2008 / 04 / 15$ & 315 & 61.85 & 1295 & 2.18 & 1.305 & 335931 \\
\hline $2008 / 04 / 16$ & 420 & 62.85 & 1715 & 3.18 & 1.314 & 306354 \\
\hline $2008 / 04 / 17$ & 374 & 63.75 & 2089 & 4.08 & 1.322 & 291607 \\
\hline $2008 / 04 / 18$ & 420 & 64.85 & 2509 & 5.18 & 1.332 & 276686 \\
\hline $2008 / 04 / 19$ & 280 & 64.35 & 2789 & 4.68 & 1.328 & 336779 \\
\hline $2008 / 04 / 20$ & 475 & 65.15 & 3264 & 5.48 & 1.335 & 336582 \\
\hline $2008 / 04 / 22$ & 315 & 65.85 & 3964 & 6.18 & 1.341 & 361225 \\
\hline $2008 / 04 / 23$ & 455 & 66.85 & 4419 & 7.18 & 1.350 & 347226 \\
\hline $2008 / 04 / 24$ & 385 & 66.85 & 4804 & 7.18 & 1.350 & 376094 \\
\hline $2008 / 04 / 25$ & 210 & 67.05 & 5014 & 7.38 & 1.352 & 381648 \\
\hline
\end{tabular}

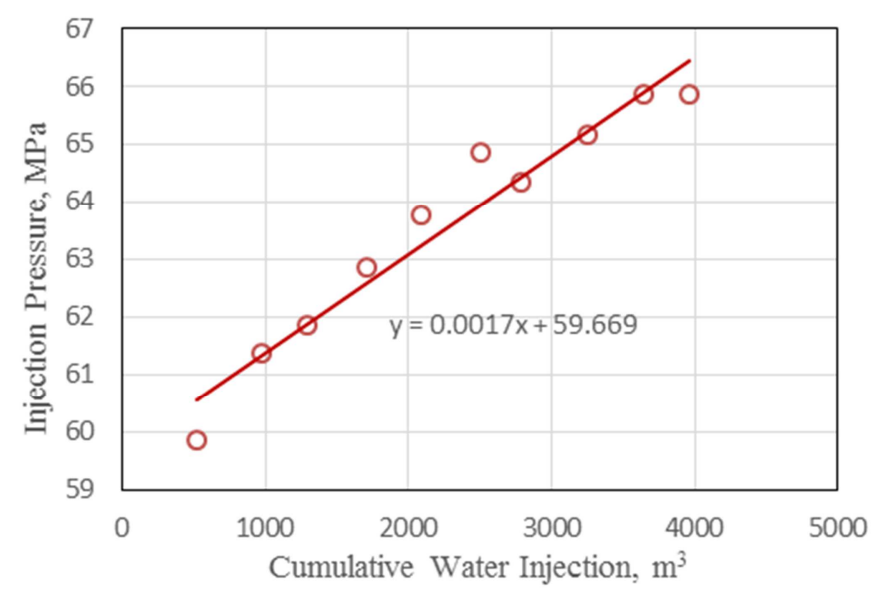

Figure 5. Conventional Water Injection Index Curve of T6-433CX. 


\subsection{Use of the Improved Water Injection Index Curve}

Based on the new technique in section 2.2, a new plot of oil in place $(N)$ vs cumulative water injection $\left(V_{\mathrm{w}}\right)$ of T6-433CX is drawn, as shown in Figure 6. The oil and gas production and fluid properties are list in Table 2, the water injection data and relevant calculated results are as shown in Table 1

From the $N$ versus $V_{\text {w }}$ plot in Figure 6, it is noted that the oil in place $N$ is approximately near to a constant horizontal line during the water injection process. The average value of $N$ is $24.7 \times 10^{4} \mathrm{~m}^{3}$, which is much low than that from the previous conventional water injection index curve, showing that the influence of secondary gas cap on the calculation of oil in place cannot be neglected. Meanwhile the volumetric OIP of $10.47 \times 10^{4} \mathrm{~m}^{3}$ was given in 2010 , the cumulative oil production at that time was $7.5 \times 10^{4} \mathrm{~m}^{3}$, and now this well has been replaced by $\mathrm{T} 6-433 \mathrm{CX} 2$ in the same area with a new total oil production of $7.2 \times 10^{4} \mathrm{~m}^{3}$, indicating that the previous volumetric OIP was a possible underestimation for this well. This, on the other hand, increases the reliability of the OIP from the improved water injection index curve method.

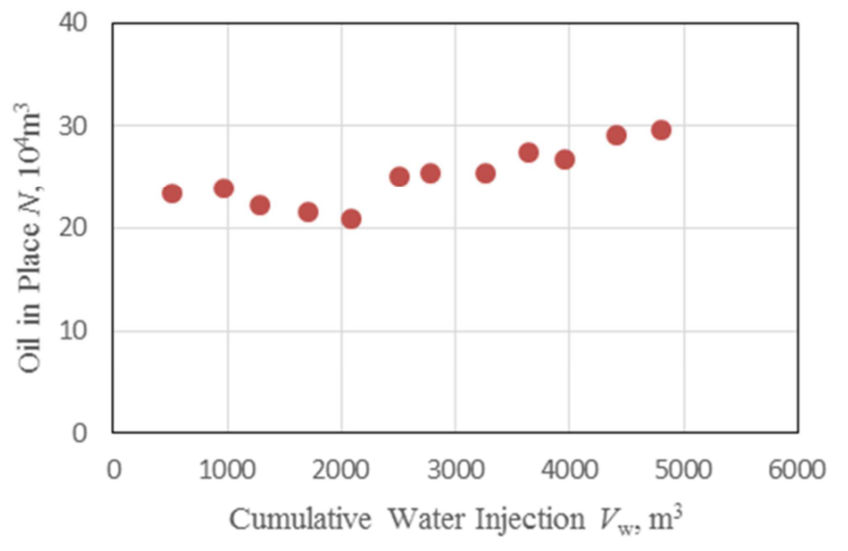

Figure 6. Improved Water Injection Index Curve of T6-433CX.

Table 2. Production Data and Fluid Properties of Well T6-433CX.

\begin{tabular}{llll}
\hline Parameters & Values & Parameters & Values \\
\hline Cumulative Production, $\mathrm{m}^{3}$ & 26346 & Reservoir Temp., ${ }^{\circ} \mathrm{C}$ & 142 \\
Cumulative Gas Oil Ratio, $\mathrm{m}^{3} / \mathrm{m}^{3}$ & 39.248 & Initial Solution Gas Ratio, $\mathrm{m}^{3} / \mathrm{m}^{3}$ & 75 \\
Oil Compressibility, $1 / \mathrm{MPa}$ & 0.0013 & Current Solution Gas Ratio, $\mathrm{m}^{3} / \mathrm{m}^{3}$ & 40 \\
\hline
\end{tabular}

Finally the improved water injection index curve method has advantage over some production index based curves [14] in conversion of well-head pressure to well-bottom pressure due to the single phase flow in the well bore. It provides an efficient new approach on OIP evaluation, avoiding dealing with the high uncertainty of aquifers in carbonate reservoir.

\section{Conclusions}

An improved water injection index curve is presented for oil in place estimation of fractured vuggy carbonate reservoirs taking into consideration the impact of secondary gas cap. When using this technique in oilfield practice:

1. Avoid using the very early water injection data until the water phase filled the wellbore completely.

2. The estimated OIP is highly sensitive to fluid properties and bottom-hole pressure, so make sure the data and parameters reliable and accurate.

3. Ignoring the impact of second gas cap compressibility leads to over-estimation of OIP, so results verification between different methods are necessary and important.

4. Bottom-hole pressure is needed when using improved water injection index curve method and the conversion of well-head to well-bottom pressure is easy to accomplish due to the single phase flow in the well bore.

\section{Nomenclature}

$B_{g}=$ gas formation volume factor

$B_{o}=$ oil formation volume factor
$C_{o}=$ oil compressibility, $1 / \mathrm{MPa}$

$N=$ Original oil in place (OOIP), $\mathrm{m}^{3}$

$N_{p}=$ cumulative oil production, $\mathrm{m}^{3}$

$p, p^{\prime}=$ reservoir pressure, $\mathrm{MPa}$

$P_{S C}=$ surface standard pressure, $\mathrm{MPa}$

$R_{p}=$ production gas ratio at $p, \mathrm{sm}^{3} / \mathrm{m}^{3}$

$R_{s i}=$ initial solution gas ratio, $\mathrm{sm}^{3} / \mathrm{m}^{3}$

$R_{s}=$ solution gas ratio at $p, \mathrm{sm}^{3} / \mathrm{m}^{3}$

$P_{S C}=$ surface standard pressure, $\mathrm{MPa}$

$V_{o}=$ reservoir oil volume at $p, \mathrm{~m}^{3}$

$V_{o}{ }^{\prime}=$ reservoir oil volume at $p^{\prime}, \mathrm{m}^{3}$

$\Delta V_{o}=$ oil volume change from $p$ to $p$

$V_{g}=$ reservoir gas volume at $p, \mathrm{~m}^{3}$

$V_{g}{ }^{\prime}=$ reservoir gas volume at $p^{\prime}, \mathrm{m}^{3}$

$\Delta V_{g}=$ gas volume change from $p$ to $p^{\prime}$

$V_{w}=$ cumulative water injection, $\mathrm{m}^{3}$

$T$ =reservoir temperature, $K$

$T_{S C}=$ temperature at standard condition, $K$

$z, \mathrm{z}^{\prime}=$ deviation factor of natural gas

\section{Acknowledgements}

The author would like to thank Northwest Oilfield Branch of SINOPEC for providing the basic data of case study and acknowledge the financial support from National Science and Technology Major Project (2016ZX05033005-008). 


\section{References}

[1] Liu, X. L., Yang, J., Z. Y., and Wang Y (2006) A New Methodology on Reservoir Modelling in the Fracture-Cavity Carbonate Rock of Tah Oilfield. SPE 104429 pressented at International Oil \& Gas Conference and Exhibition in China, 5-7 December.

[2] Yangfan Li, Tao Lin, Xinyong Li et al (2008) Oil Production and Water-Cut in Natrually-Fractured Vuggy Reservoirs, SPE 115042 prestented ath the 2008 SP Russian Oil \& Gas Technical and Exhibition held in Moscow, Russia, 28-30 October.

[3] MATTAR, L., MCNEIL, R (1998) The "Flowing" Gas Material Balance, Journal of Canadian Petroleum Technology, 37 (2): $52-55$

[4] Ram G. Agarwal, David C. Gardner, Stanley W. Kleinsteiber, Del D. Fussell (1999) Analyzing Well Production Data Using Combined-Type-Curve and Decline-Curve Analysis Concepts. SPE Reservoir Eval. \& Eng. 2 (5): 478-486.

[5] MATTAR, L., ANDERSON, D. (2005) Dynamic Material Balance, CIPC 2005-113, presented at Canadian International Petroleum Conference, Calgary, Alberta, June 7-9.

[6] Li Yong, Wang Qi, Li Baozhu, Liu Zhiliang (2017) Dynamic Characterization of Different Reservoir Types for a Fractured-Caved Carbonate Reservoir. SPE 188113 presented at the SPE Kingdom of Saudi Arabia Annual Technical Symposium and Exhibition held in Dammam, Saudi Arabia, 24-27 April.

[7] SONG Hongwei, ZHANG Zhi, REN Wenbo (2012) Reserves calculation of fractured-cavity carbonate reservoirs by material balance method. Natural Gas Exploration and Development, 35 (1): 32-35.

[8] CHEN Lixin, WANG Lianshan, GAO Chunhai, et al (2016) A new method to calculate dynamic reserves in fractured-vuggy reservoirs: a case from Halahatang oilfield, Tarim basin. Xinjiang Petroleum Geology, 37 (3): 356-359.
[9] WANG Ping, PAN Wenqing, LI Shiyin, et al (2017) Using well production performance to identify cave-cleft bodies in fractured-vuggy carbonate reservoirs: a case study of Ha-6 well block in Halahatang oilfield. Xinjiang Petroleum Geology, 38 (3): 363-368.

[10] LI Yong, YU Qingyan, LI Baozhu et al (2017) Quantitative evaluation method of OOIP and aquifer size for fractured-caved carbonate reservoirs with active aquifer support. Scientia Sinica Technologica, 47 (7): 708-717.

[11] ZHENG Songqing, CUI Shuyue, MU Lei (2018) Material balance equation and driving energy analysis of fracture-cave oil reservoir, Special Oil and Gas Reservoirs, 25 (1): 64-67.

[12] Jin Yonghong, Li Anguo1, Jie Hui, Tian Jing and Hu Lijuan (2013) Application of waterflood index curve in carbonate reservoirs, RESERVOIR EVALUATION AND DEVELOPMENT, 3 (4): 30-34.

[13] Mei Shengwen, Chen Xiaofan, Yue Ping, Tang Chao, Yi Hu (2015) An Improved New Model of Water Injection Index Curve Theory for Fractured and Caved Carbonate Reservoir. Journal of Yangtze University (Natural Science Edition), 12 (29): 57-62.

[14] YANG Meihua, ZHONG Haiquan, LI Yingchuan (2020) New production index curve of fractured-vuggy carbonate reservoirs $[\mathrm{J} / \mathrm{OL}]$, LITHOLOGIC RESERVOIRS, https://kns.cnki.net/kcms/detail/62.1195.TE.20200728.0936.0 04.html.

[15] LI Hongboa, WANG Cuilib, NIU Gea, LIANG Hongtaoa, BU Lulua, GU Junyinga (2020) Dynamic Reserves Evaluation of Fractured-Cavity Reservoirs With Closed Water: A Case From Halahatang Oilfield, Tarim Basin, XINJIANG PETROLEUM GEOLOGY, 41 (3): 321-325.

[16] ZHANG B Y, CHEN X F, YUE P. (2020) Research on unit mining by elastic drive of fractured-vuggy carbonate reservoir with bottom water by water intrusion. Reservoir Evaluation and Development, 10 (2): 71-75. 